\title{
Is arrhythmogenicity related to the speed of reperfusion during thrombolysis for myocardial infarction?
}

\author{
V. Gressin, A. P. M. Gorgels*, Y. Louvard, H. Lardoux and R. H. Bigelow $\dagger$ \\ Cardiology Department, Gilles de Corbeil Hospital, Corbeil-Essonnes, France, ${ }^{*}$ Cardiology Department, Academic \\ Hospital, Maastricht, The Netherlands and †Biometrics Department, Du Pont Pharma, Geneva, Switzerland
}

KEY WORDS: Acute myocardial infarction, thrombolytic therapy, Holter recording, ventricular arrhythmias, ST segment, reperfusion.

\begin{abstract}
The objective of this study was to relate the number of ventricular arrhythmias (VA) to the normalization time of the $S T$ segment during thrombolysis for acute myocardial infarction. The $24 \mathrm{~h} \mathrm{Holter} \mathrm{recordings,} \mathrm{begun} \mathrm{on} \mathrm{start} \mathrm{of} \mathrm{intravenous}$ thrombolytic therapy, and the 12-lead electrocardiograms of 41 patients with a patent infarct-related artery according to coronary angiography were analysed. The mean time from onset of chest pain to angiography was $30 \cdot 5 \pm 3 \cdot 1 \mathrm{~h}, \leqslant 24 \mathrm{~h}$ in $59 \%$. The normalization time of the ST segment, assessed by the time of decrease of ST segment elevation from start of Holter recording to normal or steady state was $\leqslant 60$ min in 13 patients (group 1), 60 to 180 min in 15 patients (group 2) and $>180$ min in 13 patients ( $g r o u p 3$ ). The incidence of $V A$ was similar in all groups, except for ventricular tachycardias $(V T)>15$ beats (group $1: 69 \%$, group $2: 13 \%$, group $3: 15 \%, \mathrm{P}=0.002$ ). The frequency of accelerated idioventricular rhythms $(A I V R)$, early $A I V R(\leqslant 6 \mathrm{~h})$ and of VT was significantly higher in group 1 than in group 3 with a 8-, 30- and 6fold increase, respectively (back transformed mean). We conclude that the number of $V A s$ is related to the normalization time of the ST segment during reperfusion. This may suggest that faster reflow is more arrhythmogenic.
\end{abstract}

\begin{abstract}
Introduction
Ventricular arrhythmias and ST segment changes are non-invasive signs of reperfusion during thrombolytic therapy for acute myocardial infarction ${ }^{[1.2]}$. The frequency of occurrence of ventricular arrhythmias and the time course of ST segment normalization vary between patients with successful reperfusion. The latter may suggest differences in the speed of reperfusion. We have investigated the relationship between ventricular arrhythmias and the course of ST segment normalization, the latter as a possible expression of the speed of reperfusion of the infarct-related artery.
\end{abstract}

\section{Methods \\ STUDY PATIENTS}

Forty-eight consecutive patients hospitalized for transmural acute myocardial infarction within $8 \mathrm{~h}$ of onset of chest pain, who had no contra-indication to thrombolytic therapy and who had given their informed consent were included in the study. Intravenous thombolytic treatment consisted of streptokinase ( 1.5 million units in $45 \mathrm{~min}$ ) or of recombinant tissue-type plasminogen activator (70 to $100 \mathrm{mg}$ in 90 to $180 \mathrm{~min}$ after a bolus of $10 \mathrm{mg}$ ). Administration of heparin was systematically begun at the end of infusion of the thrombolytic agent. No beta-blocker or calcium-channel antagonist was administered before or together with thrombolytic treatment. After publication of the results of the Second International Study of Infarct Survival ${ }^{[3]}$, the last 14 patients received an intravenous Submitted for publication on 6 March 1992, and in revised form 27 August 1992.

Correspondence Virginie Gressin, MD, Cardiology Department (Dr Lardoux). Gilles de Corbeil Hospital, 59 boulevard Henri Dunant, 91100 Corbeil-Essonnes, France. bolus of $250 \mathrm{mg}$ of aspirin on start of the infusion of thrombolytic treatment. No preventive antiarrhythmic treatment was given.

Twelve- or 15-lead electrocardiograms were recorded on a simultaneous 3-lead Mingocard 4 electrocardiographic recorder (Siemens-Elema) at the beginning of thrombolytic treatment and at $0 \cdot 25,0 \cdot 5,0 \cdot 75,1,1 \cdot 5,2,3$, $6,12,24$ and $48 \mathrm{~h}$ after start of the infusion. Continuous electrocardiographic monitoring was performed on a RCM 32 (ELA Medical) monitor.

Blood samples for the determination of plasma levels of creatine kinase were obtained on start of the infusion of thrombolytic treatment and 1, 2, 3, 4, 8, 12, 24 and $48 \mathrm{~h}$ after the beginning of infusion.

Twenty-four-hour two-channel Holter recording was performed using an ELA 2448 analogic Holter recorder with amplitude modulation and a recording speed of $1 \mathrm{~mm} \cdot \mathrm{s}^{-1}$ (ELA Medical). Holter recording began as soon as possible after admission to the hospital and the tapes were analysed on a digital system (Laser Holter, Marquette Electronics). Each morphological pattern (normal and ventricular complexes) was validated beat after beat. Rhythmic events with the validated ventricular complexes were then defined as isolated ventricular premature complexes, couplets or runs of $\geq$ three beats. Each ventricular run was individually inspected in all patients and then studied for its length and rate. The rate of a run was classified according to the two shortest consecutive RR intervals. All rhythmic events were studied on an hourly basis. The ST segment algorithm used incremental averaging. Each normal QRS complex was compared with a median template.

Coronary angiography was performed as part of the protocol in all patients. The infarct-related artery grade flow 
was assessed by two blinded independent observers according to the criteria defined in the Thrombolysis in Myocardial Infarction trial ${ }^{[4]}$. Only the subgroup of patients in whom the infarct-related artery was found to be patent (grade 2 or 3 ) were included in the analysis. Left ventricular ejection fraction was calculated from the right anterior oblique angiographic view according to Dodge's method. Coronary artery diameters were evaluated with a caliper. The mean of the pre- and post-stenosis diameters was used to evaluate residual stenosis of the infarct-related artery.

The following parameters were considered: the ST segment changes were evaluated from Holter recordings (two-lead printings with an amplitude of $1 \mathrm{mV} . \mathrm{cm}^{-1}$ and a recording speed of $25 \mathrm{~mm} . \mathrm{s}^{-1}$ and computerized analysis of the ST segment). When ST segment changes were not clear from Holter recordings, they were evaluated from 12-lead electrocardiograms, in the lead with the most pronounced ST segment elevation. The normalization time of the ST segment was defined by the time of decrease from ST segment elevation on start of Holter recording to normal or to steady state $\mathrm{e}^{[5]}$. The amplitude of ST segment decrease was considered significant if $\geq 50 \%$ from initial value ${ }^{[2]}$.

The ventricular arrhythmias studied were divided into isolated and total ventricular premature complexes (isolated ventricular premature complexes plus ventricular premature complexes of all runs), accelerated idioventricular rhythms (run of $\geq$ three consecutive ventricular premature complexes at a rate $\leqslant 125$ beats $\cdot \min ^{-1}$ ), and ventricular tachycardias (run of $\geq$ three monomorphic or polymorphic consecutive ventricular premature complexes at a rate $>125$ beats min $^{-1}$ ) which were specified as prolonged ventricular tachycardia ( $>15$ beats) or rapid ventricular tachycardia ( $>200$ beats. $\mathrm{min}^{-1}$ ), and ventricular fibrillation. We have previously reported that accelerated idioventricular rhythms may occur in patients with an occluded artery, but that their onset was delayed beyond the fifth hour following the start of thrombolytic therapy. This is in contrast to patients with a patent artery in which this had already been observed in the first hour ${ }^{[6]}$. Therefore, we have specifically studied early accelerated idioventricular rhythms i.e. occurring within the first $6 \mathrm{~h}$ of recording.

\section{STATISTICAL ANALYSIS}

Quantitative data were expressed as mean \pm SEM. One-way analysis of variance was used for continuous variables. Categorical variables were compared between groups by the chi-square test. To meet the distributional assumptions for analysis of variance, arrhythmia frequencies were transformed by $\log 10(æ+1)$. Descriptive statistics include mean and standard error of frequencies in addition to back transformed mean of the log transformed data.

\section{Results}

ARRHYTHMIAS IN PATIENTS WITH A PATENT INFARCTRELATED ARTERY

Forty-one patients with a patent artery (rate of patency: $85 \%$ ) were evaluated: $83 \%$ male, mean age:
Table 1 Incidence of ventricular arrhythmias in patients with a patent artery $(n=41)$
Premature ventricular complexes

Accelerated idioventricular rhythm $\leqslant 6 \mathrm{~h}$

Ventricular tachycardia

Prolonged ventricular tachycardia

Rapid ventricular tachycardia

Ventricular fibrillation
Accelerated idioventricular rhythm
$41(100 \%)$

$37(90 \%)$

$29(71 \%)$

$32(78 \%)$

$13(32 \%)$

$10(24 \%)$

$1(2 \%)$
$55 \pm 1 \cdot 5$ years (range 33 to 72 ), $37 \%$ with anterior infarction, $12 \%$ with prior myocardial infarction. The first 10 patients $(24 \%)$ were treated with streptokinase and the following $31(76 \%)$ were given recombinant tissue-type plasminogen activator. Mean delay from chest pain to thrombolysis was $3.3 \pm 0.2 \mathrm{~h}$ (range 0.75 to 7.25 ). The mean time from onset of chest pain to the beginning of Holter recording was $3.2 \pm 0.2 \mathrm{~h}$ (range 0.67 to 7 ). Holter monitoring began before thrombolysis in $83 \%$ of the patients. The mean duration of recording was $23.8 \pm 0.4 \mathrm{~h}$ (range 11.6 to 25.5 ). Coronary angiography was performed with a mean delay from the start of chest pain of $30.5 \pm 3.1 \mathrm{~h}$ (range 5 to 75 ); in 24 cases $(59 \%$ ), this was within the first $24 \mathrm{~h}$.

All patients had ventricular arrhythmias (Table 1). Tolerance was good even in the presence of a prolonged or a rapid ventricular arrhythmia. Cardioversion was required only once for ventricular fibrillation starting with an $\mathrm{R}$ on $\mathrm{T}$ phenomenon occurring before ST segment decrease. No arrhythmic death occurred during the study.

\section{ARRHYTHMIAS AND ST SEGMENT}

Three groups of patients were defined according to the normalization time of the ST segment: $\leqslant 60 \mathrm{~min}$ (group 1; $\mathrm{n}=13$ ), 60 to $180 \mathrm{~min}$ (group $2 ; \mathrm{n}=15$ ), >180 $\min$ (group $3 ; n=13$ ). No significant statistical differences were found between the three groups with regard to baseline clinical characteristics (Table 2).

The prevalence (expressed as percentage of patients with $\geq$ one episode) of the ventricular arrhythmias that we have considered was not statistically different in the three groups, except for prolonged ventricular tachycardia, the incidence of which was higher in group 1 than in groups 2 and 3: 69, 13 and $15 \%$, respectively $(P=0.002)$. The average frequency of the ventricular arrhythmias in the three groups is listed in Table 3 . All arrhythmias were more frequent in group 1 than in group 2 and than in group 3. The frequency of accelerated idioventricular rhythms, early accelerated idioventricular rhythms and ventricular tachycardias was significantly higher in group 1 than in group 3, with a 8-, 30- and 6-fold increase, respectively when back transformed means were considered. The increased frequency of ventricular tachycardias included a higher number of prolonged ventricular tachycardias, as well as of short lasting ventricular tachycardias. The time course of the hourly total frequency of accelerated idioventricular rhythms and ventricular tachycardias throughout the $24 \mathrm{~h}$ of recording in groups 
Table 2 Clinical characteristics of patients according to the normalization time of the ST segment

\begin{tabular}{|c|c|c|c|}
\hline ST segment normalization time & $\begin{array}{c}\text { Group } 1(n=13) \\
\leqslant 60 \mathrm{~min}\end{array}$ & $\begin{array}{l}\text { Group } 2(n=15) \\
\quad 60-180 \mathrm{~min}\end{array}$ & $\begin{array}{c}\text { Group } 3(\mathrm{n}=13) \\
>180 \mathrm{~min}\end{array}$ \\
\hline Sex ( $\%$ males $)$ & $11(85 \%)$ & $11(73 \%)$ & $12(92 \%)$ \\
\hline Age (years) & $55 \pm 3$ & $54 \pm 3$ & $58 \pm 2$ \\
\hline Infarct location (\% anterior) & $4(31 \%)$ & $4(27 \%)$ & $7(54 \%)$ \\
\hline Thrombolysis (\% streptokinase) & $5(38 \%)$ & $3(20 \%)$ & $2(15 \%)$ \\
\hline Aspirn (\% patients on aspirin) & $3(23 \%)$ & $7(47 \%)$ & $4(44 \%)$ \\
\hline Delay from pain to thrombolysis $(\mathrm{h})$ & $3 \cdot 0 \pm 0 \cdot 5$ & $3 \cdot 3 \pm 0 \cdot 3$ & $3 \cdot 5 \pm 0 \cdot 3$ \\
\hline Delay from pain to peak creatune kinase (h) & $15 \cdot 2 \pm 16$ & $16 \cdot 7 \pm 14$ & $15 \cdot 5 \pm 1 \cdot 7$ \\
\hline Delay from thrombolysis to peak creatine kınase (h) & $12 \cdot 2 \pm 17$ & $13 \cdot 4 \pm 1 \cdot 2$ & $12 \cdot 1+1 \cdot 7$ \\
\hline Peak creatine kinase $\left(I U .1^{-1}\right)$ & $2750 \pm 526$ & $2277 \pm 401$ & $2262 \pm 419$ \\
\hline Delay from pain to angiography $(h)$ & $32 \cdot 4 \pm 6.3$ & $29.4 \pm 4.9$ & $29 \cdot 7 \pm 5 \cdot 5$ \\
\hline Delay from pain to Holter $(h)$ & $3.0+0.5$ & $3 \cdot 1+0 \cdot 3$ & $3.4+0.4$ \\
\hline Holter duration $(\mathrm{h})$ & $235 \pm 0.9$ & $24 \cdot 0 \pm 0.9$ & $23.9 \pm 0.4$ \\
\hline Ejection fraction (\%) & $55 \pm 4$ & $59 \pm 3$ & $54 \pm 5$ \\
\hline Residual stenosis (\%) & $70 \pm 4$ & $68 \pm 6$ & $63+7$ \\
\hline
\end{tabular}

All results NS

Table 3 Frequency of ventricular arrhythmias according to the normalization time of the ST segment

\begin{tabular}{|c|c|c|c|c|c|c|c|}
\hline \multirow{2}{*}{$\begin{array}{l}\text { ST segment normalization time } \\
\text { Single premature ventricular complexes }\end{array}$} & \multicolumn{2}{|c|}{$\begin{array}{c}\text { Group } 1(n=13) \\
\leqslant 60 \mathrm{~min}\end{array}$} & \multicolumn{2}{|c|}{$\begin{array}{l}\text { Group } 2(\mathrm{n}=15) \\
\quad 60-180 \mathrm{~min}\end{array}$} & \multicolumn{2}{|c|}{$\begin{array}{l}\text { Group } 3(n=13) \\
\quad>180 \mathrm{~min}\end{array}$} & \multirow{2}{*}{$\begin{array}{l}P \\
\mathrm{NS}\end{array}$} \\
\hline & $1428 \pm 475$ & $(660)$ & $1091+351$ & $(574)$ & $404 \pm 104$ & $(28 \mathrm{I})$ & \\
\hline Total premature ventricular complexes & $5925+1786$ & $(1819)$ & $1926+718$ & (911) & $596+146$ & $(406)$ & $<0.05^{* *}$ \\
\hline Couplets & $168 \pm 57$ & (49) & $65 \pm 27$ & (29) & $25 \pm 7$ & (14) & NS \\
\hline Accelerated idioventricular rhythm & $258 \pm 84$ & (46) & $66 \pm 31$ & (19) & $22 \pm 14$ & (6) & $0.05^{* *}$ \\
\hline Accelerated idioventricular rhythm $\leqslant 6 h$ & $174 \pm 52$ & (30) & $22 \pm 13$ & (4) & $5 \pm 3$ & (1) & $0.005^{*}$ \\
\hline Ventricular tachycardia & $74+45$ & (12) & $9 \pm 6$ & (3) & $4+1$ & (2) & $0.02^{*}$ \\
\hline Prolonged ventricular tachycardia & $12 \pm 7$ & (3) & $1 \cdot 3 \pm 1 \cdot 2$ & $(0 \cdot 3)$ & $0 \cdot 2 \pm 0 \cdot 1$ & $(0 \cdot 1)$ & $0.006^{*}$ \\
\hline Short lasting ventricular tachycardia & $61 \pm 38$ & (11) & $7 \pm 5$ & (3) & $4 \pm 1$ & (2) & $0.03 * *$ \\
\hline Rapid ventricular tachycardia & $3 \pm 2$ & (1) & $0.8 \pm 0.7$ & $(0 \cdot 2)$ & $0.5 \pm 0.3$ & $(0 \cdot 3)$ & NS \\
\hline
\end{tabular}

Mean $\pm S E M$ (back transformed mean), ${ }^{*} 1$ versus 2 and 1 versus $3,{ }^{* *} 1$ versus 3 .

$P$ values are for $l o g$ tranformed data.

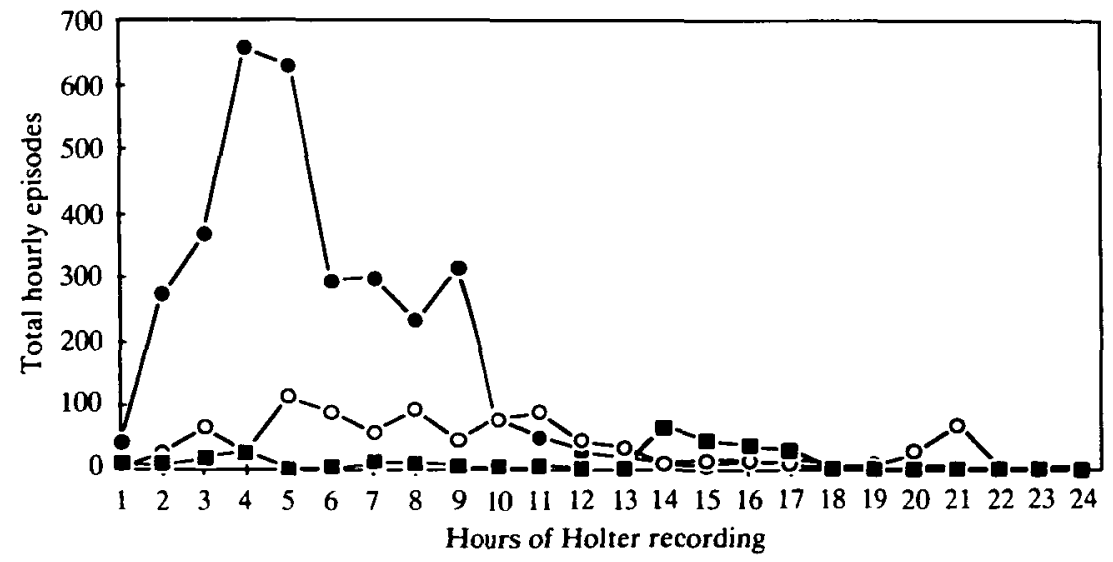

Figure 1 Hourly average frequency of accelerated idioventricular rhythm according to the normalization time of the ST segment. $=$ group 1; $\mathrm{O}=$ group 2; $\mathbf{\square}=$ group 3 .

1,2 and 3 are shown in Figs 1 and 2, respectively. These figures show a very high frequency of accelerated idioventricular rhythms and ventricular tachycardias in group 1 during the early hours after start of Holter recordings. Moreover, those arrhythmias occur early, with a peak of ventricular tachycardias during the second hour of 


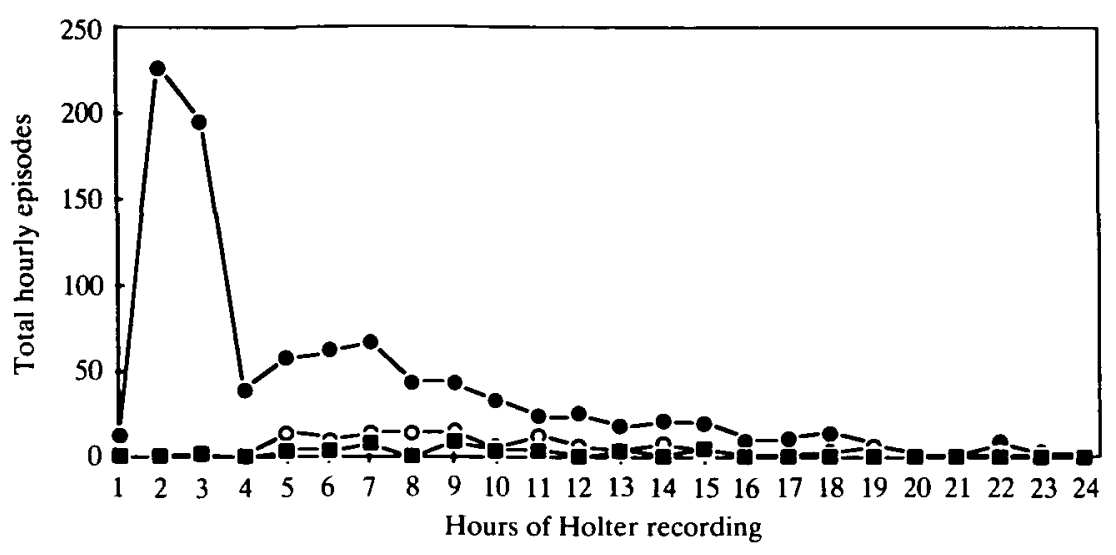

Figure 2 Hourly average frequency of ventricular tachycardia according to the normalization time of the ST segment. = group $1 ; \mathrm{O}=$ group $2 ; \boldsymbol{\square}=$ group 3 .

recording and a slightly delayed peak of accelerated idioventricular rhythms during the fourth hour. The frequency of both arrhythmias is very low beyond the tenth hour of recording.

\section{Discussion}

Studies with Holter recordings and continuous electrocardiographic monitoring have shown that ventricular arrhythmias are frequent and well tolerated during thrombolysis ${ }^{[6-8]}$. Coronary artery patency is associated with an increase in incidence ${ }^{[8-10]}$ and/or frequency ${ }^{[6,11]}$ of ventricular tachycardias and accelerated idioventricular rhythms. Several authors have tried to identify factors which may influence this arrhythmogenicity of reperfusion. Usual clinical parameters, such as age, infarct location, gender, the thrombolytic agent used (streptokinase or recombinant tissue-type plasminogen activator), the interval from onset of chest pain to thrombolysis and reperfusion were not found to influence the prevalence and frequency of ventricular arrhythmias ${ }^{[6,8,9]}$. In addition, no correlation was demonstrated between the number of runs of accelerated idioventricular rhythm and peak creatine kinase activity, serum potassium and creatinine, sinus rate and number of diseased vessels ${ }^{[6,9,11]}$; only Cercek et $a l^{[8]}$ found a significant positive correlation between the maximal initial ST segment elevation ( $<$ or $>2 \mathrm{~mm}$ ) and frequency of accelerated idioventricular rhythm.

Currently, routine thrombolysis is based on intravenous rather than intracoronary administration of fibrinolytic agents and coronary angiography is not performed in the acute phase in most of the cases. It may still be required, particularly when thrombolysis has failed to achieve patency. The need to identify reperfusion has led to the search for non-invasive criteria, such as chest pain relief ${ }^{[1.8]}$, ST segment changes and/or ventricular arrhythmias ${ }^{[1,2,5,6,8,9,11,12]}$ or creatine kinase or myoglobin kinetics $^{[13-15]}$. Angiographic studies have demonstrated a fall in ST segment concomitant with the restoration of antegrade flow ${ }^{[7]}$ and a chronological relationship between the latter and onset of ventricular arrhythmias ${ }^{[7]}$.
The best criterion (in terms of sensitivity and specificity for the diagnosis of reperfusion) of ST segment change has not yet been clearly established ${ }^{[2,5,11]}$.

Our hypothesis is that the normalization time of the ST segment is related to the speed of reperfusion. If this is correct, then our observation of a higher frequency of ventricular arrhythmias in the group where the normalization time of the ST segment is short in comparison to groups where it lasts longer suggests that fast reperfusion is more arrhythmogenic than slow reperfusion. This is consistent with the low frequency of ventricular arrhythmias that we had previously observed in non-reperfused patients ${ }^{[6]}$. It is interesting to note that the two arrhythmias the incidence or frequency of which is highly significantly linked to the normalization time of the ST segment are prolonged ventricular tachycardias and early accelerated idioventricular rhythms. All ventricular arrhythmias considered, those two arrhythmias were the best noninvasive criteria for coronary patency in our previous work $^{[6]}$.

In man, the potential deleterious effect of rapid reperfusion has been suggested by some authors. Particularly, a residual stenosis of $80 \%$ or less was found to be a predisposing factor for the occurrence of ventricular fibrillation, ventricular tachycardia and accelerated idioventricular rhythm at reperfusion by Maras $e t a l^{[16]}$ in a study of intracoronary thrombolysis with acute documentation of coronary patency. Because of the lower risk of ventricular fibrillation observed in dogs with a flow-limiting partial stenosis of the artery undergoing transient occlusion than in dogs with normal coronary arteries ${ }^{[17]}$, it was assumed that the rate of reflow was higher when the residual stenosis was less severe and that it may play a role in the arrhythmogenicity of reperfusion ${ }^{[16]}$. We did not find any difference in residual stenosis between the three groups. This may suggest that the speed of reperfusion is dependent on other factors than residual stenosis.

In animal models, a short duration of ischaemia or a rapid speed of reperfusion have been demonstrated to precipitate the onset of reperfusion arrhythmias ${ }^{[17-19]}$ but the respective role of the status of the arrhythmogenic 
substrate (degree of ischaemia) or of the quality of the trigger (the reperfusate composition) ${ }^{[18,19]}$ in the arrhythmogenicity of reperfusion may be different depending on the rate or on the delay of reperfusion. Sudden reflow leads to abrupt washout of toxic metabolites or reoxygenation; this contributes to tissue heterogeneity and rapid electrical instability leading to arrhythmias ${ }^{[18.19]}$. Several metabolic changes due to ischaemia and/or reperfusion, such as calcium overload, production of free radicals, high fatty acid levels, impaired potassium and magnesium concentrations have been thought to play a role in the arrhythmogenicity of reperfusion ${ }^{[18]}$. It has been reported that the incidence of reperfusion arrhythmias is decreased when the speed of reperfusion is reduced, possibly because of slower and more uniform washout of toxic metabolites ${ }^{[18]}$. These biochemical factors might accelerate the cellular damage due to ischaemia and may thus be responsible for 'reperfusion injury' ${ }^{\text {'20] }}$. A recent study has shown that staged reperfusion attenuates myocardial stunning and that this beneficial effect is primarily attributable to transient acidosis during early reperfusion. Calcium overload as well as activation of leucocytes and free-radical generation, which can be attenuated by acidosis, have been reported to cause myocardial stunning ${ }^{[21]}$. We may assume that the higher incidence of arrhythmias observed when reperfusion is suspected to be more abrupt is indicative of a more severe myocardial damage. We did not find any difference in acute ejection fraction between the three groups, but ejection fraction was not assessed again on a second occassion such as 1 month after the acute phase; this could have provided an insight on myocardial recovery. A prospective study is underway to evaluate this interesting issue.

Our study is limited by the small size of the population and the lack of a more accurate method for the evaluation of the speed of reperfusion. Furthermore, the relationship between the frequency of occurrence of acute arrhythmias and prognostic factors remains to be established. Attempts to modify the reperfusate composition, such as administration of free-radical scavengers or angiotensin converting enzyme inhibitors have succeeded in reducing the incidence of reperfusion arrhythmias in animals ${ }^{[18.22]}$. If arrhythmias may be considered as a marker for reperfusion injury, their analysis may be important during studies aimed to improve the quality of reperfusion in $\operatorname{man}^{[23]}$.

\section{References}

[1] Kircher BJ, Topol EJ, O'Neill WW, Pitt B. Prediction of infarct coronary artery recanalization after intravenous thrombolytic therapy. Am J Cardiol 1987; 59: 513-5.

[2] Hohnloser SH, Zabel M, Kasper W, Meinertz T, Just H. Assessment of coronary artery patency after thrombolytic therapy: accurate prediction utilizing the combined analysis of three noninvasive markers. J Am Coll Cardiol 1991; 18: 44-9.

[3] ISIS 2 (Second International Study of Infarct Survival) Collaborative Group. Randomised trial of intravenous streptokinase, oral aspirin, both, or neither among 17187 cases of suspected acute myocardial infarction: ISIS 2. Lancet 1988; ii: 34960
[4] Chesebro JH, Knatterud G, Roberts R et al. Thrombolysis in Myocardial Infarction (TIMI) trial, phase 1: a comparison between intravenous tissue plasminogen activator and intravenous streptokinase. Clinical findings through hospital discharge. Circulation 1987; 76: 142-54.

[5] Krucoff MW, Green CE, Satler LF et al. Non invasive detection of coronary artery patency using continuous ST segment monitoring. Am J Cardiol 1986; 57. 916-22.

[6] Gressin V, Louvard Y, Pezzano M, Lardoux H. Holter recording of ventricular arrhythmias during intravenous thrombolysis for acute myocardial infarction. Am J Cardiol 1992; 69: 152-9.

[7] Goldberg S, Greespon AJ, Urban PL et al. Reperfusion arrhythmias: a marker of restoration of anterograde flow during intracoronary thrombolysis for acute myocardial infarction. Am Heart J 1983; 105: 26-32.

[8] Cercek B, Lew AS, Laramee P, Shah PK, Peter TC, Ganz W. Time course and characteristics of ventricular arrhythmias after reperfusion in acute myocardial infarction. Am J Cardiol 1987; 60: 214-8.

[9] Gorgels APM, Vos MA, Letsch IS et al. Usefulness of the accelerated idioventricular rhythm as a marker for myocardial necrosis and reperfusion during thrombolytic therapy in acute myocardial infarction. Am J Cardiol 1988; 61: 231-5.

[10] Miller FC, Krucoff MW, Satler LF et al. Ventricular arrhythmias during reperfusion. Am Heart J 1986; 112: 928-32.

[11] Zehender M, Utzolino S, Furtwangler A, Kasper W, Meinertz $T$, Just $H$. Time course and interrelation of reperfusion-induced ST changes and ventricular arrhythmias in acute myocardial infarction. Am J Cardiol 1991; 68: 1138-42.

[12] Doevendans PA, Gorgels APM, Wellens HJ. Electrocardiographic signs of reperfusion in acute myocardial infarction Circulation 1991; II-117 (Abstr).

[13] Lewis BS, Ganz W, Laramee P, Cercek B, Hod H, Shah PK, Lew AS. Usefulness of a rapid inttial uncrease in plasma creatine kinase activity as a marker of reperfusion during thrombolytic therapy for acute myocardial infarction. Am J Cardiol 1988; 62: 20-4.

[14] Grande P, Granborg J, Clemmensen P, Sevilla DC, Wagner NB, Wagner GS. Indices of reperfusion in patients with acute myocardial infarction using characteristics of the CK-MB curve. Am Heart J 1991; 122: 400-8.

[15] Ellis AK, Little T, Masud ARZ, Klocke FJ. Patterns of myoglobin release after reperfusion of injured myocardium Circulation 1985; 72: 639-47.

[16] Maras P, Della Grazia E, Klugmann S et al. Reperfusion ventricular arrhythmias during intracoronary thrombolysis. Eur Heart J 1986; 7A: 23-30.

[17] Sheehan F, Epstein SE. Determinants of arrhythmic death due to coronary spasm: effect of preexisting coronary artery stenosis on the incidence of reperfusion arrhythmia. Circulation 1982; 65: $259-64$.

[18] Manning AS, Hearse DJ. Reperfusion-induced arrhythmias: mechanisms and prevention. J Mol Cell Cardiol 1984; 16: 497-518.

[19] Corr PB, Witkowski FX. Potential electrophysiologic mechanisms responsible for dysthythmias associated with reperfusion of ischemic myocardium. Circulation 1983; 68: 16-24.

[20] Nayler WG, Elz JS. Reperfusion injury: laboratory artifact or clinical dilemma? Circulation 1986; 74: 215-21.

[21] Hori M, Kitakaze M, Sato $\mathrm{H}$ et al. Staged reperfusion attenuates myocardial stunning in dogs. Role of transient acidosis during early reperfusion. Circulation 1991; 84: 2135-45.

[22] Van Gilst WH, De Graeff PA, Wesseling H, De Langen CDJ Reduction of reperfusion arrhythmias in the ischemic isolated rat heart by angiotensin converting enzyme inhibitors: a comparison of captopril, enalapril and HOE 498. J Cardiovasc Pharmacol 1986; 8: 722-8.

[23] Van Gilst WH, Kingma JH (CATS Investigators Group). Early intervention with angiotensin-converting enzyme inhibitors during thrombolytic therapy in acute myocardial infarction: rationale and design of captopril and thrombolysis study. Am J Cardiol 1991: 68: D111 5. 